Research Article

\title{
Extension on Bifurcations of Traveling Wave Solutions for a Two-Component Fornberg-Whitham Equation
}

\author{
Zhenshu Wen \\ School of Mathematical Sciences, Huaqiao University, Quanzhou 362021, China \\ Correspondence should be addressed to Zhenshu Wen, zhenshu.wen@mail.scut.edu.cn
}

Received 20 September 2012; Accepted 16 November 2012

Academic Editor: Zhenya Yan

Copyright (C) 2012 Zhenshu Wen. This is an open access article distributed under the Creative Commons Attribution License, which permits unrestricted use, distribution, and reproduction in any medium, provided the original work is properly cited.

Fan et al. studied the bifurcations of traveling wave solutions for a two-component FornbergWhitham equation. They gave a part of possible phase portraits and obtained some uncertain parametric conditions for solitons and kink (antikink) solutions. However, the exact explicit parametric conditions have not been given for the existence of solitons and kink (antikink) solutions. In this paper, we study the bifurcations for the two-component Fornberg-Whitham equation in detalis, present all possible phase portraits, and give the exact explicit parametric conditions for various solutions. In addition, not only solitons and kink (antikink) solutions, but also peakons and periodic cusp waves are obtained. Our results extend the previous study.

\section{Introduction}

In 2011, Fan et al. [1] introduced the following two-component Fornberg-Whitham equation

$$
\begin{gathered}
u_{t}=u_{x x t}-u_{x}-u u_{x}+3 u_{x} u_{x x}+u u_{x x x}+\rho_{x} \\
\rho_{t}=-(\rho u)_{x^{\prime}}
\end{gathered}
$$

where $u=u(x, t)$ denotes the height of the water surface above a horizontal bottom, and $\rho=\rho(x, t)$ indicts the horizontal velocity field. They studied the bifurcations of traveling wave solutions for (1.1) through obtaining some uncertain parametric conditions for solitons, kink (antikink) solutions, and further gave some expressions of those solutions. However, they did not give the explicit parametric conditions for the existence of solitons and kink (antikink) solutions. In this paper, we further analyze the bifurcations for (1.1) systematically 
by exploiting the bifurcation method and qualitative theory of dynamical systems [2-7]. We present all possible phase portraits determinately and give all the exact explicit parametric conditions for various solutions. Additionally, we obtain explicit peakons and periodic cusp waves for (1.1), which were not included in [1].

\section{Bifurcations of Phase Portraits}

In this section, we will present the process of obtaining the bifurcations of phase portraits for (1.1). follows,

For given constant $c$, substituting $u(x, t)=\varphi(\xi), \rho=\psi(\xi)$ with $\xi=x-c t$ into (1.1), it

$$
\begin{gathered}
-c \varphi^{\prime}+c \varphi^{\prime \prime \prime}+\varphi^{\prime}+\varphi \varphi^{\prime}-3 \varphi^{\prime} \varphi^{\prime \prime}-\varphi \varphi^{\prime \prime \prime}-\psi^{\prime}=0, \\
-c \psi^{\prime}+\varphi^{\prime} \psi+\varphi \psi^{\prime}=0,
\end{gathered}
$$

where the prime stands for derivative with respect to the variable $\xi$.

Integrating (2.1) once leads to

$$
\begin{gathered}
(1-c) \varphi+\frac{1}{2} \varphi^{2}-\left(\varphi^{\prime}\right)^{2}+(c-\varphi) \varphi^{\prime \prime}-\psi=g \\
(\varphi-c) \psi=G
\end{gathered}
$$

where both $g$ and $G$ are integral constants.

From the second equation of system (2.2), we obtain

$$
\psi=\frac{G}{\varphi-c}
$$

Substituting (2.3) into the first equation of system (2.2), it follows:

$$
(1-c) \varphi+\frac{1}{2} \varphi^{2}-\left(\varphi^{\prime}\right)^{2}+(c-\varphi) \varphi^{\prime \prime}-\frac{G}{\varphi-c}=g .
$$

By setting $\varphi=\phi+c-2 / 3,(2.4)$ becomes

$$
\left(\frac{2}{3}-\phi\right)^{2} \phi^{\prime \prime}=\left(\frac{2}{3}-\phi\right)\left(\phi^{\prime}\right)^{2}+\frac{1}{2} \phi^{3}-\frac{1}{6}\left(3 c^{2}-6 c+4+6 g\right) \phi+\frac{1}{27}\left(9 c^{2}-18 c+8+18 g\right)-G
$$


Letting $y=\phi^{\prime}$, we obtain a planar system

$$
\begin{gathered}
\frac{d \phi}{d \xi}=y, \\
\frac{d y}{d \xi}=\frac{(2 / 3-\phi) y^{2}+(1 / 2) \phi^{3}-(1 / 6)\left(3 c^{2}-6 c+4+6 g\right) \phi+(1 / 27)\left(9 c^{2}-18 c+8+18 g\right)-G}{(2 / 3-\phi)^{2}},
\end{gathered}
$$

with first integral

$$
H(\phi, y)=\left(\frac{2}{3}-\phi\right)^{2} y^{2}-\frac{1}{4} \phi^{4}+\frac{1}{6}\left(3 c^{2}-6 c+4+6 g\right) \phi^{2}-\frac{2}{27}\left(9 c^{2}-18 c+8+18 g\right) \phi+2 G \phi
$$

Note that when $G=0$, system (2.6) and (2.7) become

$$
\begin{gathered}
\frac{d \phi}{d \xi}=y, \\
\frac{d y}{d \xi}=\frac{y^{2}-(1 / 2) \phi^{2}-(1 / 3) \phi+(1 / 6)\left(3 c^{2}-6 c+4+6 g\right)-2 / 9}{2 / 3-\phi}, \\
H(\phi, y)=\left(\frac{2}{3}-\phi\right)^{2} y^{2}-\frac{1}{4} \phi^{4}+\frac{1}{6}\left(3 c^{2}-6 c+4+6 g\right) \phi^{2}-\frac{2}{27}\left(9 c^{2}-18 c+8+18 g\right) \phi,
\end{gathered}
$$

respectively.

Transformed by $d \xi=(2 / 3-\phi)^{2} d \tau$, system (2.6) becomes a Hamiltonian system

$$
\begin{gathered}
\frac{d \phi}{d \xi}=\left(\frac{2}{3}-\phi\right)^{2} y \\
\frac{d y}{d \xi}=\left(\frac{2}{3}-\phi\right) y^{2}+\frac{1}{2} \phi^{3}-\frac{1}{6}\left(3 c^{2}-6 c+4+6 g\right) \phi+\frac{1}{27}\left(9 c^{2}-18 c+8+18 g\right)-G
\end{gathered}
$$

Since the first integral of system (2.6) is the same as that of the Hamiltonian system (2.10), system (2.6) should have the same topological phase portraits as system (2.10) except the straight line $l: \phi=2 / 3$. Therefore, we should be able to obtain the topological phase portraits of system (2.6) from those of system (2.10).

Let

$$
f(\phi)=\frac{1}{2} \phi^{3}-\frac{1}{6}\left(3 c^{2}-6 c+4+6 g\right) \phi+\frac{1}{27}\left(9 c^{2}-18 c+8+18 g\right)-G
$$

It is easy to obtain the two extreme points of $f(\phi)$ as follows:

$$
\phi_{ \pm}^{*}= \pm \frac{1}{3} \sqrt{3 c^{2}-6 c+4+6 g}, \quad \text { for } g>-\frac{1}{2}\left(c^{2}-2 c+\frac{4}{3}\right),
$$


from which we can obtain a critical curve for $g$ as follows:

$$
g_{0}(c)=-\frac{1}{2}\left(c^{2}-2 c+\frac{4}{3}\right)
$$

Further, we obtain two bifurcation curves:

$$
\begin{aligned}
& G_{1}=\frac{1}{27}\left[\left(3 c^{2}-6 c+4+6 g\right) \sqrt{3 c^{2}-6 c+4+6 g}+9 c^{2}-18 c+8+18 g\right] \\
& G_{2}=\frac{1}{27}\left[-\left(3 c^{2}-6 c+4+6 g\right) \sqrt{3 c^{2}-6 c+4+6 g}+9 c^{2}-18 c+8+18 g\right]
\end{aligned}
$$

from $f\left(\phi_{-}^{*}\right)=0$ and $f\left(\phi_{+}^{*}\right)=0$, respectively. Note that when $g>g_{0}(c)$, obviously $G_{1}>G_{2}$.

Additionally, we can obtain another two critical curves for $g$, that is,

$$
\begin{gathered}
g_{1}(c)=-\frac{1}{2}\left(c^{2}-2 c+1\right), \\
g_{2}(c)=-\frac{1}{2}\left(c^{2}-2 c\right),
\end{gathered}
$$

from $G_{1}=0$ and $G_{2}=0$, respectively.

Note that (2.16) can also be obtained by letting $\phi_{+}^{*}=2 / 3$.

Let $\left(\phi^{*}, 0\right)$ be one of the singular points of system $(2.10)$, then the characteristic values of the linearized system of system $(2.10)$ at the singular point $\left(\phi^{*}, 0\right)$ are

$$
\lambda_{ \pm}= \pm \sqrt{\left(\frac{2}{3}-\phi^{*}\right)^{2} f^{\prime}\left(\phi^{*}\right)}
$$

From the qualitative theory of dynamical systems, we can determine the property of singular point $\left(\phi^{*}, 0\right)$ by the sign of $f^{\prime}\left(\phi^{*}\right)$.

Based on the above analysis, we give the information of the singular points for system (2.10) and their relationship with $\phi_{-}^{*}, \phi_{+}^{*}$ and $2 / 3$ when $g>g_{2}(c)$, as an illustration, in the following lemma.

Lemma 2.1. For $g>g_{2}(c)$, one has $G_{1}<0<G_{2}$ and the singular points of system (2.10) can be described as follows.

(a) If $G<G_{2}$, then there is only one singular point denoted as $S_{1}\left(\phi_{1}, 0\right)\left(\phi_{1}<\phi_{-}^{*}<2 / 3<\right.$ $\left.\phi_{+}^{*}\right) . S_{1}$ is a saddle point.

(b) If $G=G_{2}$, then there are two singular points denoted as $S_{1}\left(\phi_{1}, 0\right)$ and $S_{2}\left(\phi_{2}, 0\right)\left(\phi_{1}<\right.$ $\left.\phi_{-}^{*}<2 / 3<\phi_{+}^{*}=\phi_{2}\right)$, respectively. $S_{1}$ is a saddle point and $S_{2}$ is a degenerate saddle point.

(c) If $G_{2}<G<0$, then there are three singular points denoted as $S_{1}\left(\phi_{1}, 0\right), S_{2}\left(\phi_{2}, 0\right)$, and $S_{3}\left(\phi_{3}, 0\right)\left(\phi_{1}<\phi_{-}^{*}<2 / 3<\phi_{2}<\phi_{+}^{*}<\phi_{3}\right)$, respectively. $S_{1}$ and $S_{3}$ are saddle points and $S_{2}$ is a center. 

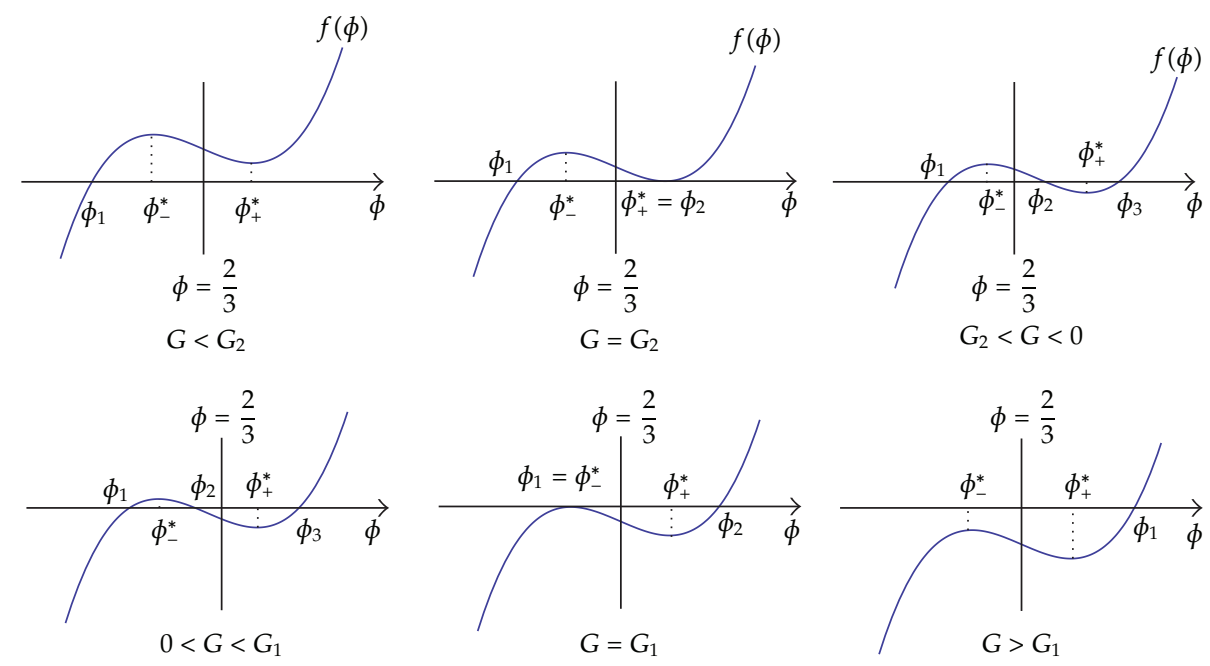

Figure 1: The graphics of $f(\phi)$ when $g>g_{2}(c)$.

(d) If $0<G<G_{1}$, then there are three singular points denoted as $S_{1}\left(\phi_{1}, 0\right), S_{2}\left(\phi_{2}, 0\right)$, and $S_{3}\left(\phi_{3}, 0\right)\left(\phi_{1}<\phi_{-}^{*}<\phi_{2}<2 / 3<\phi_{+}^{*}<\phi_{3}\right)$, respectively. $S_{1}$ and $S_{3}$ are saddle points and $S_{2}$ is a center.

(e) If $G=G_{1}$, then there are two singular points denoted as $S_{1}\left(\phi_{1}, 0\right)$ and $S_{2}\left(\phi_{2}, 0\right)\left(\phi_{1}=\right.$ $\left.\phi_{-}^{*}<2 / 3<\phi_{+}^{*}<\phi_{2}\right)$, respectively. $S_{1}$ is a degenerate saddle point and $S_{2}$ is a saddle point.

(f) If $G>G_{1}$, then there is only one singular point denoted as $S_{1}\left(\phi_{1}, 0\right)\left(\phi_{-}^{*}<2 / 3<\phi_{+}^{*}<\right.$ $\left.\phi_{1}\right) . S_{1}$ is a saddle point.

Proof. Lemma 2.1 follows easily from the graphics of the function $f(\phi)$, which can be obtained directly and shown in Figure 1 (note that $f(2 / 3)=G$ ).

Remark 2.2. The case when $G=0$ follows easily from the similar analysis of system (2.8), and we just omit it here for simplicity.

For the other cases, the similar analysis can be taken to make the conclusions. We just omit these processes for simplicity. However, it is worth mentioning that, when $g_{0}(c)<g<$ $g_{2}(c)$ and $G_{2}<G<\min \left(0, G_{1}\right)$, there exist two saddle points and one center lying on the same side of the singular line $l: \phi=2 / 3$. Hence, there may exist heteroclinic orbits for system (2.6). We will show the existence of heteroclinic orbits for system (2.6) under certain conditions in the following analysis.

When $G_{1}<G<G_{2}$, we set the three solutions of $f(\phi)=0$ to be $\phi_{s}, \phi_{m}$, and $\phi_{b}\left(\phi_{s}<\right.$ $\left.\phi_{m}<\phi_{b}\right)$, respectively. Through simple calculation, we can express $\phi_{s}$ and $\phi_{b}$ as the function of $\phi_{m}$, that is,

$$
\begin{aligned}
& \phi_{s}=\frac{-\phi_{m}-\sqrt{(4 / 3)\left(3 c^{2}-6 c+4+6 g\right)-3 \phi_{m}^{2}}}{2}, \\
& \phi_{b}=\frac{-\phi_{m}+\sqrt{(4 / 3)\left(3 c^{2}-6 c+4+6 g\right)-3 \phi_{m}^{2}}}{2} .
\end{aligned}
$$


It follows from $\phi_{s}<\phi_{m}<\phi_{b}$ that $\phi_{m}$ must satisfy condition

$$
\phi_{m}^{2}<\frac{3 c^{2}-6 c+4+6 g}{9} .
$$

From $H\left(\phi_{s}, 0\right)=H\left(\phi_{b}, 0\right)$, we obtain the expression of $G$ as the function of $\phi_{m}$,

$$
G=\frac{1}{8} \phi_{m}^{3}+\frac{1}{27}\left(9 c^{2}-18 c+8+18 g\right) .
$$
follows:

Substituting (2.20) into $f\left(\phi_{m}\right)=0$, we obtain the expression of $\phi_{m}$ from $f\left(\phi_{m}\right)=0$ as

$$
\begin{gathered}
\phi_{m 1}=0 \\
\phi_{m 2}=-\frac{2}{3} \sqrt{3 c^{2}-6 c+4+6 g} \\
\phi_{m 3}=\frac{2}{3} \sqrt{3 c^{2}-6 c+4+6 g} .
\end{gathered}
$$

We can easily know that (2.22) does not satisfy (2.19), while (2.21) satisfies (2.19), if $g>g_{0}(c)$ $G$ as follows:

By substituting (2.21) into (2.20), we obtain the bifurcation curve (denoted by $G^{*}$ ) for

$$
G^{*}=\frac{1}{27}\left(9 c^{2}-18 c+8+18 g\right)
$$

Note that if $g>g_{0}(c)$, then obviously we have $G_{2}<G^{*}<G_{1}$. Additionally, we have indicated that when $g_{0}(c)<g<g_{2}(c)$ and $G_{2}<G<\min \left(0, G_{1}\right)$, there exist two saddle points and one center lying on the same side of the singular line $\phi=2 / 3$. Therefore, we obtain the fourth critical curve for $g$ from $G^{*}=0$,

$$
g_{3}(c)=-\frac{1}{2}\left(c^{2}-2 c+\frac{8}{9}\right)
$$

Hence, we can express the existence of the heteroclinic orbits as follows.

Lemma 2.3. (1) For $g \in\left(g_{0}(c), g_{3}(c)\right)$ and $G=G^{*}$, there exist heteroclinic orbits for system (2.6). (2) For $g \notin\left(g_{0}(c), g_{3}(c)\right)$ or $G \neq G^{*}$, there exists no heteroclinic orbit for system (2.6).

Proof. Note that when $g_{3}(c)<g<g_{2}(c)$ (or $g=g_{3}(c)$ ), one saddle point and one center point lie on the left side of the singular line $l: \phi=2 / 3$ and the other saddle point on the right side of (or on) the singular line $l: \phi=2 / 3$. Therefore, Lemma 2.3 follows easily from the above analysis.

Thereby, based on the above analysis, we obtain the bifurcations of phase portraits for system (2.6) in Figures 2, 3, 4, 5, 6, 7, and 8 under the corresponding conditions. 


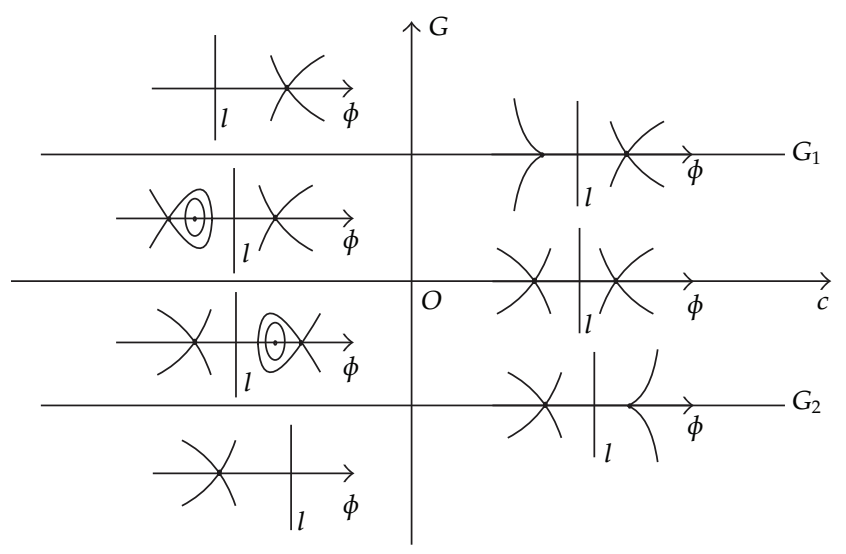

Figure 2: The phase portraits of system (2.6) when $g>g_{2}(c)$.

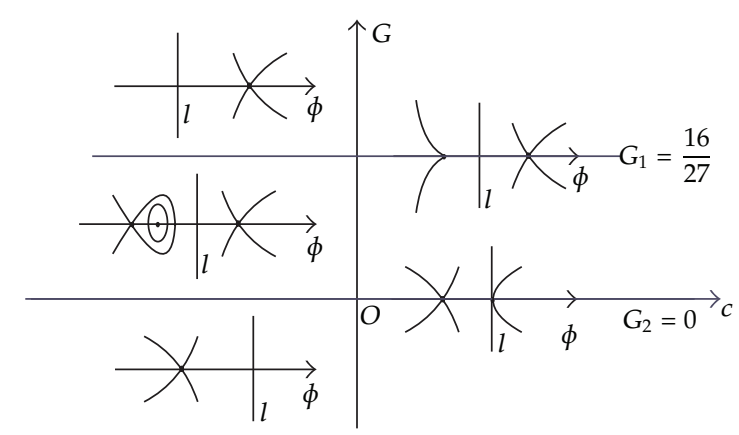

Figure 3: The phase portraits of system (2.6) when $g=g_{2}(c)$.

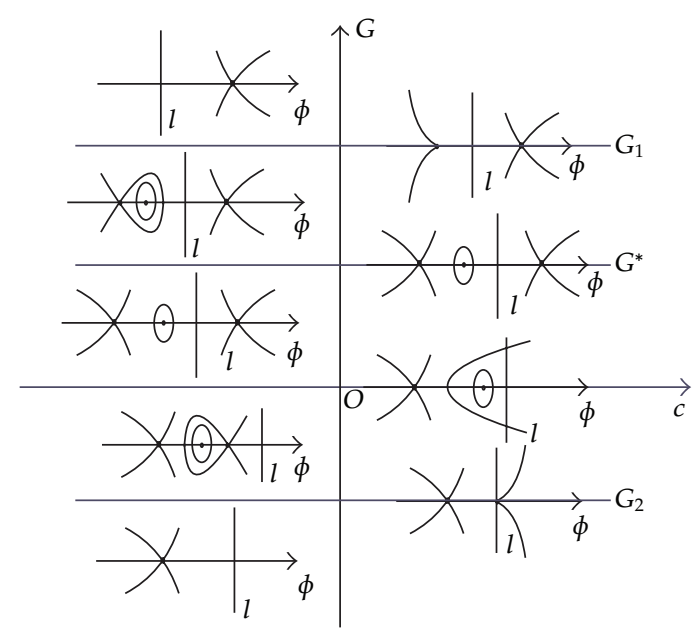

Figure 4: The phase portraits of system (2.6) when $g_{3}(c)<g<g_{2}(c)$. 


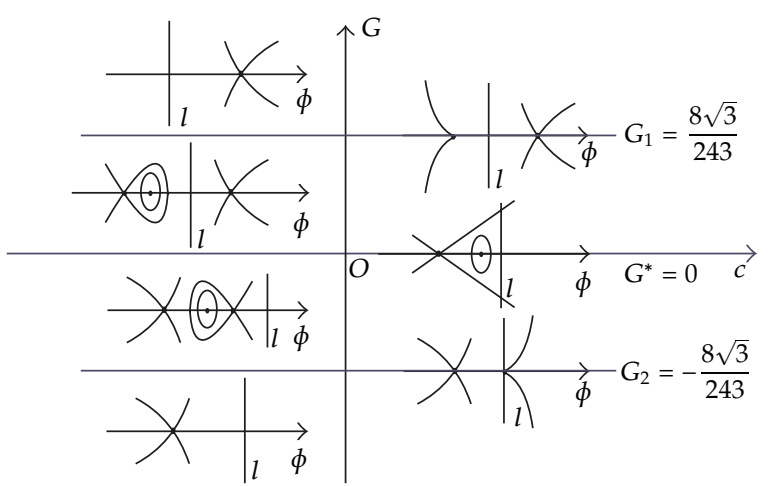

Figure 5: The phase portraits of system (2.6) when $g=g_{3}(c)$.

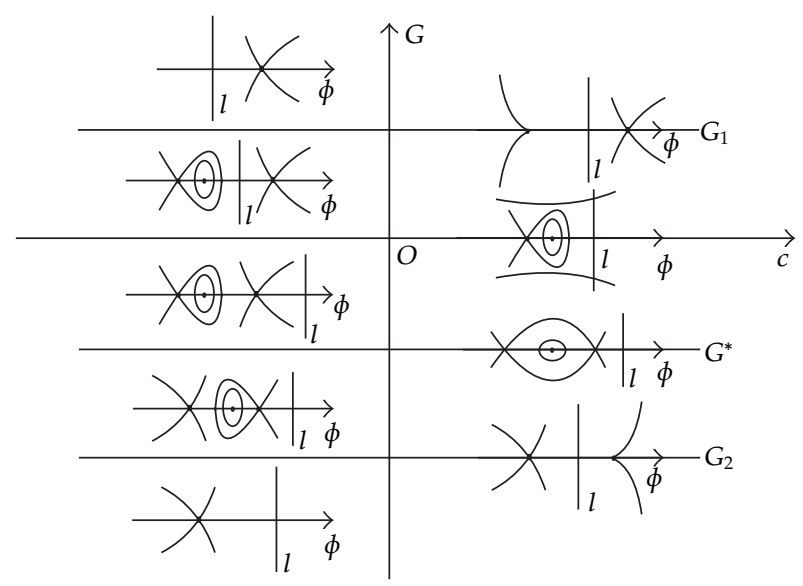

Figure 6: The phase portraits of system (2.6) when $g_{1}(c)<g<g_{3}(c)$.

\section{Main Results and the Theoretic Derivations of Main Results}

In this section, we state our results about solitons, kink (antikink) solutions, peakons, and periodic cusp waves for the first component of system (1.1). To relate conveniently, we omit $\varphi=\phi+c-2 / 3$ and the expression of the second component of system (1.1) in the following theorems.

Theorem 3.1. For constant wave speed $c$, integral constants $g$ and $G$, one has the following.

(1) If $c, g$, and $G$ satisfy one of the following conditions:

(i) $g \geq g_{2}(c)$ and $0<G<G_{1}$;

(ii) $g_{3}(c) \leq g<g_{2}(c)$ and $G^{*}<G<G_{1}$;

(iii) $g_{1}(c)<g<g_{3}(c)$ and $0 \leq G<G_{1}$;

(iv) $g_{0}(c)<g<g_{3}(c)$ and $G^{*}<G<0$, 


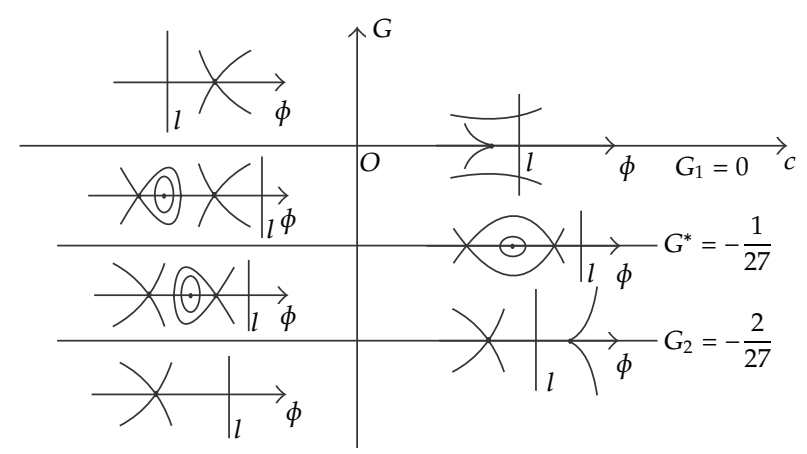

Figure 7: The phase portraits of system (2.6) when $g=g_{1}(c)$.

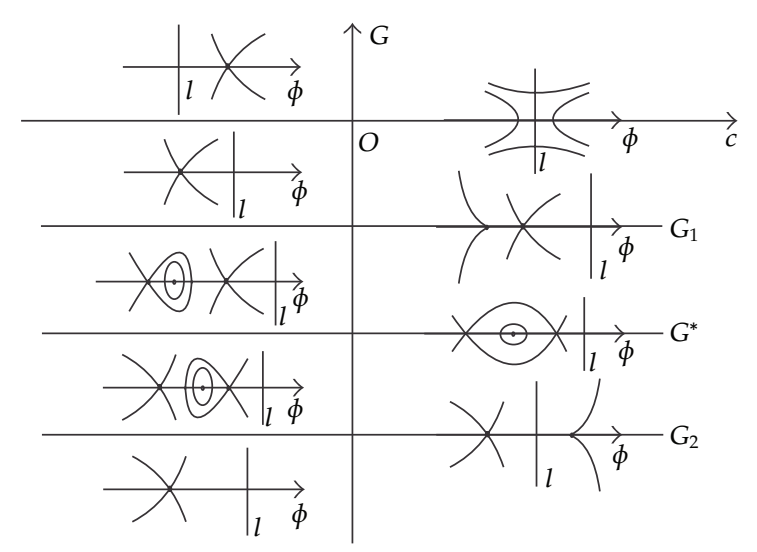

Figure 8: The phase portraits of system (2.6) when $g_{0}(c)<g<g_{1}(c)$.

then there exist soliton solutions for (1.1), which can be implicitly expressed as

$$
\begin{aligned}
& \left(2 \sqrt{\left(\phi_{12}-\phi_{1}\right)\left(\phi_{13}-\phi_{1}\right)\left(\phi_{12}-\phi\right)\left(\phi_{13}-\phi\right)}+4 \phi_{1} \phi+2 \phi_{12} \phi_{13}+2 \phi_{1}^{2}\right)^{\alpha_{1}} \\
& \quad \times\left(2 \sqrt{\left(\phi_{12}-\phi\right)\left(\phi_{13}-\phi\right)}+2 \phi+2 \phi_{1}\right) \\
& \times\left(\left(\phi-\phi_{1}\right)^{\alpha_{1}}\left(\phi_{13}-\phi_{12}\right)^{\alpha_{1}+1}\right)^{-1}=e^{|\xi| / 2},
\end{aligned}
$$

where

$$
\begin{gathered}
\phi_{12}=-\phi_{1}-\sqrt{\frac{2}{3}\left(3 c^{2}-6 c+4+6 g\right)-2 \phi_{1}^{2}}, \\
\phi_{13}=-\phi_{1}+\sqrt{\frac{2}{3}\left(3 c^{2}-6 c+4+6 g\right)-2 \phi_{1}^{2}}, \\
\alpha_{1}=\frac{2 / 3-\phi_{1}}{\sqrt{\left(\phi_{12}-\phi_{1}\right)\left(\phi_{13}-\phi_{1}\right)}} .
\end{gathered}
$$




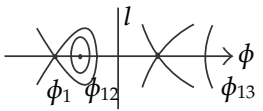

(a)

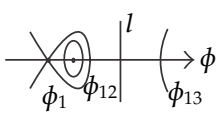

(b)

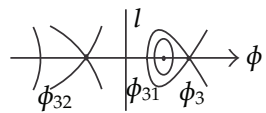

(c)

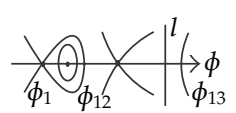

(d)

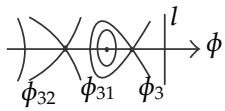

(e)

Figure 9: The different kinds of homoclinic orbits for system (2.6).

(2) If $c, g$, and G satisfy condition:

(v) $g>g_{2}(c)$ and $G_{2}<G<0$,

then there exist soliton solutions for (1.1), which can be implicitly expressed as

$$
\begin{aligned}
& \left(2 \sqrt{\left(\phi_{3}-\phi_{31}\right)\left(\phi_{3}-\phi_{32}\right)\left(\phi-\phi_{31}\right)\left(\phi-\phi_{32}\right)}+4 \phi_{3} \phi+2 \phi_{31} \phi_{32}+2 \phi_{3}^{2}\right)^{\alpha_{3}} \\
& \quad \times\left(2 \sqrt{\left(\phi-\phi_{31}\right)\left(\phi-\phi_{32}\right)}+2 \phi+2 \phi_{3}\right) \\
& \quad \times\left(\left(\phi_{3}-\phi\right)^{\alpha_{3}}\left(\phi_{31}-\phi_{32}\right)^{\alpha_{3}+1}\right)^{-1}=e^{-|\xi| / 2},
\end{aligned}
$$

where

$$
\begin{gathered}
\phi_{31}=-\phi_{3}-\sqrt{\frac{2}{3}\left(3 c^{2}-6 c+4+6 g\right)-2 \phi_{3}^{2}}, \\
\phi_{32}=-\phi_{3}+\sqrt{\frac{2}{3}\left(3 c^{2}-6 c+4+6 g\right)-2 \phi_{3}^{2}}, \\
\alpha_{3}=\frac{(2 / 3)-\phi_{3}}{\sqrt{\left(\phi_{3}-\phi_{31}\right)\left(\phi_{3}-\phi_{32}\right)}} .
\end{gathered}
$$

If $c, g$, and $G$ satisfy one of the following conditions:

(vi) $g_{3}(c) \leq g<g_{2}(c)$ and $G_{2}<G<0$;

(vii) $g_{0}(c)<g<g_{3}(c)$ and $G_{2}<G<G^{*}$,

then there exist solitons solution for (1.1), which can be implicitly expressed as

$$
\begin{aligned}
& \left(2 \sqrt{\left(\phi_{3}-\phi_{31}\right)\left(\phi_{3}-\phi_{32}\right)\left(\phi-\phi_{31}\right)\left(\phi-\phi_{32}\right)}+4 \phi_{3} \phi+2 \phi_{31} \phi_{32}+2 \phi_{3}^{2}\right)^{\alpha_{3}} \\
& \quad \times\left(2 \sqrt{\left(\phi-\phi_{31}\right)\left(\phi-\phi_{32}\right)}+2 \phi+2 \phi_{3}\right) \\
& \quad \times\left(\left(\phi_{3}-\phi\right)^{\alpha_{3}}\left(\phi_{31}-\phi_{32}\right)^{\alpha_{3}+1}\right)^{-1}=e^{|\xi| / 2},
\end{aligned}
$$

where $\phi_{31}, \phi_{32}$, and $\alpha_{3}$ are given in (3.4), (3.5), and (3.6) respectively. 
Remark 3.2. (3.1) and (3.7) are the same as those given in [1]; however, (3.3) is not shown in [1].

Remark 3.3. We give all possible homoclinic orbits in Figure 9, while it seems that Figures 9(b), 9(d), and 9(e) are not given in [1].

Proof. (1) From the phase portraits in Figures 2, 3, 4, 5, 6, 7, and 8, we see that when $c$, $g$, and $G$ satisfy one of the conditions, that is, (i), (ii), (iii), or (iv), there exist homoclinic orbits as showed individually in Figures 9(a), 9(b), or 9(d). The expressions of the homoclinic orbits can be given as follows:

$$
y= \pm \frac{\left(\phi-\phi_{1}\right) \sqrt{\left(\phi_{12}-\phi\right)\left(\phi_{13}-\phi\right)}}{2(2 / 3-\phi)}, \quad \phi_{1} \leq \phi \leq \phi_{12}<\frac{2}{3}<\phi_{13} .
$$

Substituting (3.8) into the first equation of system (2.6) and integrating along the homoclinic orbits, it follows that

$$
\int_{\phi}^{\phi_{12}} \frac{(2 / 3-s) d s}{\left(s-\phi_{1}\right) \sqrt{\left(\phi_{12}-s\right)\left(\phi_{13}-s\right)}}=\frac{1}{2}|\xi| .
$$

From (3.9), we obtain the soliton solutions (3.1).

(2) When $c, g$, and G satisfy one of the conditions, that is, (v), (vi), or (vii), there exist homoclinic orbits as showed individually in Figures 9(c) or 9(e). The expressions of the homoclinic orbits can be given as follows:

$$
y= \pm \frac{\left(\phi_{3}-\phi\right) \sqrt{\left(\phi-\phi_{31}\right)\left(\phi-\phi_{32}\right)}}{2(\phi-2 / 3)}, \quad \phi_{32}<\frac{2}{3}<\phi_{31} \leq \phi \leq \phi_{3}
$$

or

$$
y= \pm \frac{\left(\phi_{3}-\phi\right) \sqrt{\left(\phi-\phi_{31}\right)\left(\phi-\phi_{32}\right)}}{2(2 / 3-\phi)}, \quad \phi_{32}<\phi_{31} \leq \phi \leq \phi_{3}<\frac{2}{3} .
$$

Substituting (3.10) and (3.11) into the first equation of system (2.6), and integrating along the homoclinic orbits, it follows that

$$
\begin{aligned}
& \int_{\phi_{31}}^{\phi} \frac{(s-2 / 3) d s}{\left(\phi_{3}-s\right) \sqrt{\left(s-\phi_{31}\right)\left(s-\phi_{32}\right)}}=\frac{1}{2}|\xi|, \\
& \int_{\phi_{31}}^{\phi} \frac{(2 / 3-s) d s}{\left(\phi_{3}-s\right) \sqrt{\left(s-\phi_{31}\right)\left(s-\phi_{32}\right)}}=\frac{1}{2}|\xi| .
\end{aligned}
$$

From (3.12), we obtain the soliton solutions (3.3) and (3.7). 
Theorem 3.4. If integral constants $g$ and $G$ satisfy $g_{0}(c)<g<g_{3}(c)$ and $G=G^{*}$, then there exist kink and antikink solutions.

Proof. We have showed that when $g_{0}(c)<g<g_{3}(c)$ and $G=G^{*}$, there exist heteroclinic orbits for system (2.6). The heteroclinic can be expressed as

$$
y= \pm \frac{\left(\phi-\phi_{s}\right)\left(\phi_{b}-\phi\right)}{2 / 3-\phi}, \quad \phi_{s} \leq \phi \leq \phi_{b}
$$

where

$$
\begin{gathered}
\phi_{s}=-\sqrt{\frac{1}{3}\left(3 c^{2}-6 c+4+6 g\right)}, \\
\phi_{b}=\sqrt{\frac{1}{3}\left(3 c^{2}-6 c+4+6 g\right)},
\end{gathered}
$$

which can be obtained by substituting (2.23) into (2.11).

Substituting (3.13) into the first equation of system (2.6) and integrating along the heteroclinic orbits, it follows that

$$
\int_{\phi_{0}}^{\phi} \frac{(2 / 3-t) d t}{\left(t-\phi_{s}\right)\left(\phi_{b}-t\right)}= \pm \frac{1}{2} \xi
$$

where $\phi_{0} \in\left(\phi_{s}, \phi_{b}\right)$ is the initial value.

From (3.15), we have

$$
\begin{aligned}
& \left(\phi-\phi_{s}\right)^{\left(2 / 3-\phi_{s}\right) /\left(\phi_{b}-\phi_{s}\right)} \cdot\left(\phi_{b}-\phi\right)^{\left(2 / 3-\phi_{b}\right) /\left(\phi_{b}-\phi_{s}\right)} \\
& \quad=\left(\phi_{0}-\phi_{s}\right)^{\left(2 / 3-\phi_{s}\right) /\left(\phi_{b}-\phi_{s}\right)} \cdot\left(\phi_{b}-\phi_{0}\right)^{\left(2 / 3-\phi_{b}\right) /\left(\phi_{b}-\phi_{s}\right)} e^{ \pm \xi / 2} .
\end{aligned}
$$

If we take $\phi_{0}=\left(\phi_{s}+\phi_{b}\right) / 2,(3.16)$ becomes

$$
\left(\phi-\phi_{s}\right)^{\left(2 / 3-\phi_{s}\right) /\left(\phi_{b}-\phi_{s}\right)} \cdot\left(\phi_{b}-\phi\right)^{\left(2 / 3-\phi_{b}\right) /\left(\phi_{b}-\phi_{s}\right)}=\left(\frac{\phi_{b}-\phi_{s}}{2}\right)^{4 / 3 /\left(\phi_{b}-\phi_{s}\right)} e^{ \pm \xi / 2}
$$

(3.16) or (3.17) are kink (antikink) solutions.

Theorem 3.5. (1) If $g=g_{3}(c)$ and $G=0$, then there exist peakons for (1.1), which can be explicitly expressed as

$$
\phi=\frac{2}{3}\left(2 e^{-(1 / 2)|x-c t|}-1\right)
$$


(2) If $g_{3}(c)<g<g_{2}(c)$ and $G=0$, then (1.1) has periodic cusp waves

$$
u(x, t)=\phi(\xi-2 i T)+c-\frac{2}{3}
$$

where $i=0, \pm 1, \pm 2, \ldots, \xi=x-c t \in[(2 i-1) T,(2 i+1) T]$, and

$$
\begin{aligned}
\phi(\xi)= & \frac{1}{2}\left(\frac{4}{3}-\frac{1}{3} \sqrt{6\left(6 c-3 c^{2}-6 g\right)}\right) e^{(1 / 2)|x-c t|} \\
& +\frac{1}{2}\left(\frac{4}{3}+\frac{1}{3} \sqrt{6\left(6 c-3 c^{2}-6 g\right)}\right) e^{-(1 / 2)|x-c t|}-\frac{2}{3}
\end{aligned}
$$

with

$$
T=2 \ln \left(\frac{4+\sqrt{6\left(6 c-3 c^{2}-6 g\right)}}{\sqrt{2\left(9 c^{2}-18 c+8+18 g\right)}}\right) .
$$

Remark 3.6. When $G=0$, system (2.8) is the exact planar system of the Fornberg-Whitham equation $[8,9]$, and peakons (3.18) and periodic cusp waves (3.19) are the same as those in [9].

Proof. (1) When $g=g_{3}(c)$ and $G=0$, from Figure 5, we see that there is a triangle orbit, which can be expressed as

$$
\begin{gathered}
y= \pm \frac{1}{2}\left(\phi+\frac{2}{3}\right), \quad \text { for }-\frac{2}{3} \leq \phi \leq \frac{2}{3}, \\
\phi=\frac{2}{3}, \quad \text { for }-\frac{2}{3} \leq y \leq \frac{2}{3} .
\end{gathered}
$$

Substituting (3.22) into the first equation of system (2.6) and integrating along the triangle orbits, it follows that

$$
\int_{\phi}^{2 / 3} \frac{d t}{s+2 / 3}=\frac{1}{2}|\xi|
$$

From (3.24), we obtain peakons (3.18).

(2) When $g_{3}(c)<g<g_{2}(c)$ and $G=0$, from Figure 4, we see that there is an semiellipse orbit, which can be expressed as

$$
\begin{gathered}
y= \pm \frac{1}{2} \sqrt{\phi^{2}+\frac{4}{3} \phi+\frac{4}{3}-\frac{2}{3}\left(3 c^{2}-6 c+4+6 g\right)}, \quad \text { for } \frac{1}{3}\left(\sqrt{2\left(9 c^{2}-18 c+8+18 g\right)}-2\right) \leq \phi \leq \frac{2}{3} \\
\phi=\frac{2}{3}, \quad \text { for }-\frac{1}{6} \sqrt{6\left(6 c-3 c^{2}-6 g\right)} \leq y \leq \frac{1}{6} \sqrt{6\left(6 c-3 c^{2}-6 g\right)} .
\end{gathered}
$$


Substituting (3.25) into the first equation of system (2.6) and integrating along the semiellipse orbit, it follows that

$$
\int_{\phi}^{2 / 3} \frac{d t}{\sqrt{\phi^{2}+(4 / 3) \phi+(4 / 3)-(2 / 3)\left(3 c^{2}-6 c+4+6 g\right)}}=\frac{1}{2}|\xi| .
$$

From (3.27), we obtain periodic cusp waves (3.19) along with (3.20) and (3.21).

Remark 3.7. Our results are more general than those in [10] from the perspective of parameters, because the results in [10] are obtained by setting integral constant to a special value $\left(h=(3 c / 32)\left(c^{2}-8 g\right)\right.$ in [10]), while our results are obtained under all possible parametric conditions.

Remark 3.8. The system investigated in this paper is relatively complicated for all parameters. That is why Fan et al. [1] did not show the exact parametric conditions for kink (antikink) solutions. However, we figure out a new way to obtain the exact explicit parametric conditions for kink (antikink) solutions and obtain solitons under more general conditions.

\section{Conclusions}

Based on a previous paper [1], we further study the bifurcations of traveling wave solutions for the two-component Fornberg-Whitham equation, present all possible phase portraits determinately, and show all the exact explicit parametric conditions under which there exist solitons and kink (or antikink) solutions for (1.1). In addition, we obtain peakons and periodic cusp waves with explicit expressions for (1.1). Our results extend the previous study [1], which only gives some possible phase portraits, some undetermined parametric conditions, and some implicit expressions of solitons and kink (or antikink) solutions.

\section{Acknowledgment}

This research is supported by Huaqiao University (no. 12BS223).

\section{References}

[1] X. Fan, S. Yang, J. Yin, and L. Tian, "Bifurcations of traveling wave solutions for a two-component Fornberg-Whitham equation," Communications in Nonlinear Science and Numerical Simulation, vol. 16, no. 10, pp. 3956-3963, 2011.

[2] Z. Liu and C. Yang, "The application of bifurcation method to a higher-order KdV equation," Journal of Mathematical Analysis and Applications, vol. 275, no. 1, pp. 1-12, 2002.

[3] Z. Liu and J. Li, "Bifurcations of solitary waves and domain wall waves for KdV-like equation with higher order nonlinearity," International Journal of Bifurcation and Chaos in Applied Sciences and Engineering, vol. 12, no. 2, pp. 397-407, 2002.

[4] M. Song, S. Li, and J. Cao, "New exact solutions for the (2+1)-dimensional Broer-Kaup-Kupershmidt equations," Abstract and Applied Analysis, vol. 2010, Article ID 652649, 9 pages, 2010.

[5] Z. Wen, Z. Liu, and M. Song, "New exact solutions for the classical Drinfel'd-Sokolov-Wilson equation," Applied Mathematics and Computation, vol. 215, no. 6, pp. 2349-2358, 2009.

[6] Z. Wen and Z. Liu, "Bifurcation of peakons and periodic cusp waves for the generalization of the Camassa-Holm equation," Nonlinear Analysis: Real World Applications, vol. 12, no. 3, pp. 1698-1707, 2011. 
[7] Z. Wen, "Bifurcation of traveling wave solutions for a two-component generalized $\theta$-equation," Mathematical Problems in Engineering. In press.

[8] J. Zhou and L. Tian, "A type of bounded traveling wave solutions for the Fornberg-Whitham equation," Journal of Mathematical Analysis and Applications, vol. 346, no. 1, pp. 255-261, 2008.

[9] J. Zhou and L. Tian, "Solitons, peakons and periodic cusp wave solutions for the Fornberg-Whitham equation," Nonlinear Analysis: Real World Applications, vol. 11, no. 1, pp. 356-363, 2010.

[10] J. Zhou, L. Tian, and X. Fan, "Soliton, kink and antikink solutions of a 2-component of the DegasperisProcesi equation," Nonlinear Analysis: Real World Applications, vol. 11, no. 4, pp. 2529-2536, 2010. 


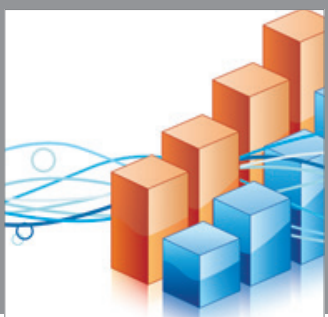

Advances in

Operations Research

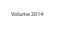

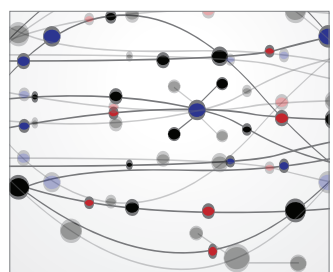

\section{The Scientific} World Journal
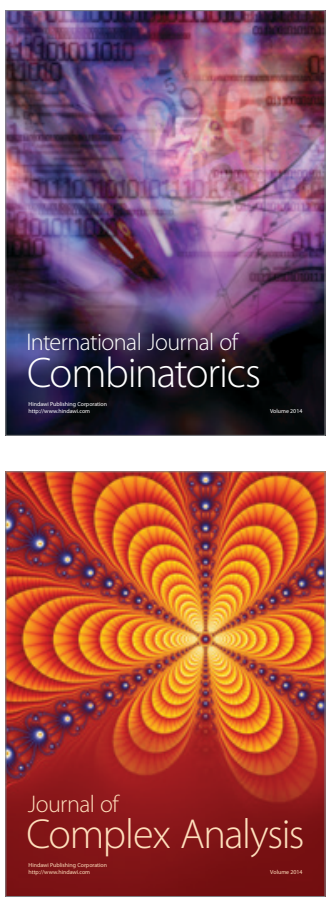

International Journal of

Mathematics and

Mathematical

Sciences
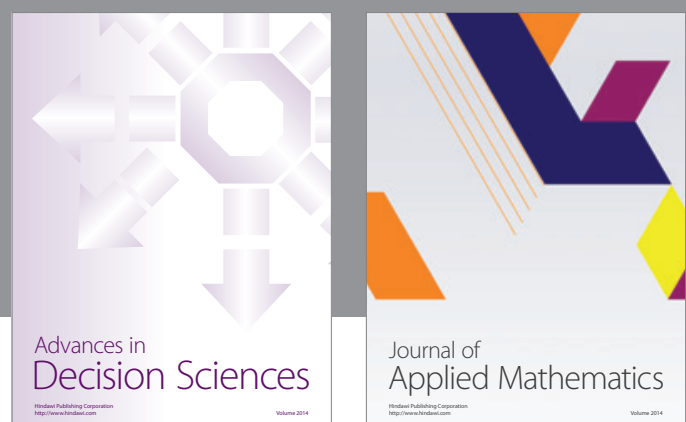

Journal of

Applied Mathematics
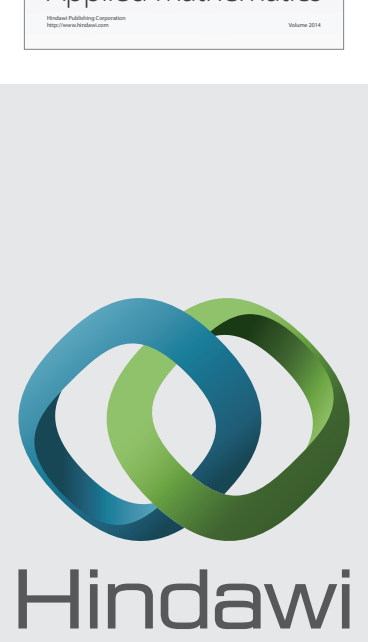

Submit your manuscripts at http://www.hindawi.com
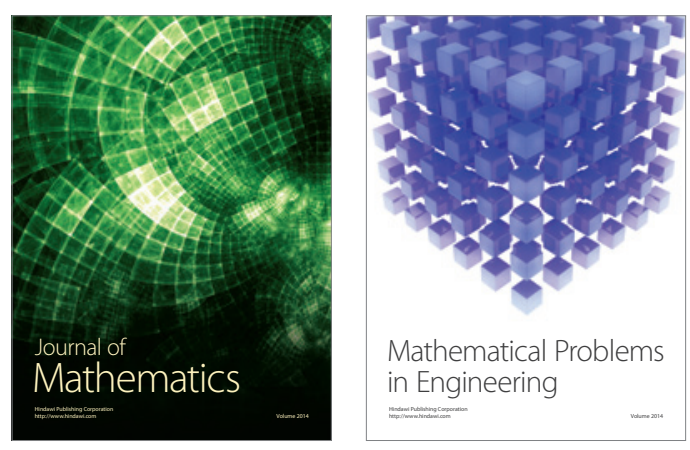

Mathematical Problems in Engineering
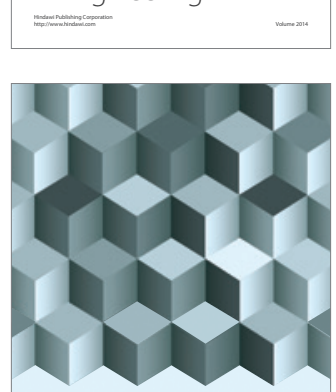

Journal of

Function Spaces
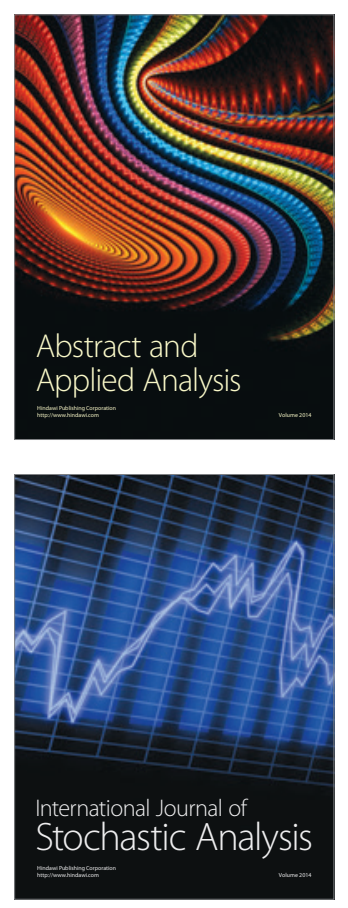

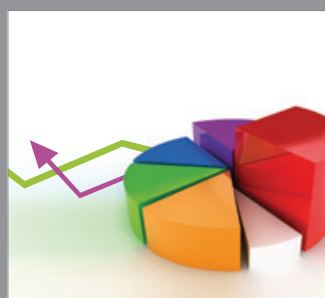

ournal of

Probability and Statistics

Promensencen
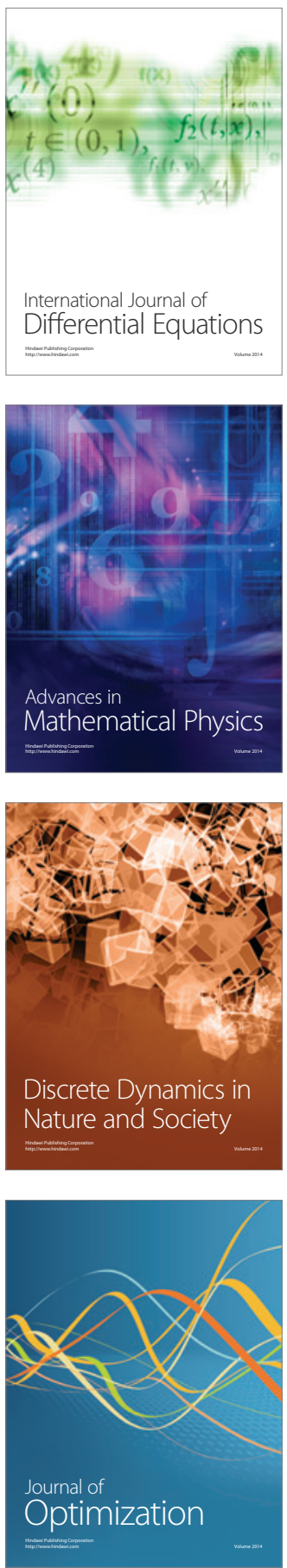